\title{
A new family of $S U(2)$ symmetric integrable sigma models
}

\author{
J. Balog, P. Forgács \\ Research Institute for Particle and Nuclear Physics \\ H-1525 Budapest 114, P. O. B. 49, Hungary \\ Z. Horváth, L. Palla \\ Institute for Theoretical Physics \\ Roland Eötvös University \\ H-1088 Budapest, Puskin u. 5-7, Hungary
}

\begin{abstract}
Local Lagrangeans are derived for a class of $S U(2)$ invariant sigma models admitting two commuting Kac-Moody algebras at the level of Poisson brackets. The one loop renormalizability of these models is established. Some heuristic arguments are presented in favour of their quantum integrability.
\end{abstract}


In this paper we study a special class of two dimensional sigma models which admit two Poisson bracket (PB) commuting Kac-Moody algebras (KMA's). To consider such models has been suggested some time ago by Rajeev [1] whose motivation was to establish a direct connection between the principal chiral sigma model (P $\Sigma \mathrm{M})$ and the chiral Gross-Neveu (or non-abelian Thirring) model. This approach might be useful to provide an alternative quantization of the P $\Sigma \mathrm{M}$, see refs. $[2,3]$ for the 'standard' methods.

One of our main result is a family of local Lagrangeans depending on a 'deformation' parameter (in addition to the usual coupling) providing a realization of the canonical PB's corresponding to the commuting KMA's. Our Lagrangean describes a deformation of the $\mathrm{P} \Sigma \mathrm{M}$ in the sense that for some value of the deformation parameter it yields the standard eqs. of motion and PB's for the currents, however, it cannot be transformed to the known one by local field redefinitions.

We also show that the deformed Lagrangeans are renormalizable at the one loop level and provide some heuristic arguments about their quantum integrability.

Let us start with the standard equations of motion written in terms of currents valued in the Lie algebra of a semisimple Lie group $G, I_{\mu}=\mathrm{i} \lambda^{a} I_{\mu}^{a}$ : *

$$
\begin{aligned}
& \partial_{\mu} I^{\mu}=0 \\
& \partial_{\mu} I_{\nu}-\partial_{\nu} I_{\mu}+\left[I_{\mu}, I_{\nu}\right]=0
\end{aligned}
$$

As it is well known eqs. $(1 \mathrm{a}, 1 \mathrm{~b})$ are the compatibility conditions of an auxiliary linear problem [4]:

$$
\left[\mathcal{D}_{\mu}(\lambda), \mathcal{D}_{\nu}(\lambda)\right]=0 \quad \mathcal{D}_{\mu}(\lambda)=\partial_{\mu}+\frac{\lambda^{2}}{\lambda^{2}-1} I_{\mu}+\frac{\lambda}{\lambda^{2}-1} \epsilon_{\mu \rho} I^{\rho}
$$

hence they admit an infinite number of (classically) conserved quantities. Most known two dimensional integrable models correspond to eqs. (1) or to some of their reductions.

The direct application of the quantum inverse scattering methods [5] to eqs. (1) has been severely hampered by the non-ultralocality of many interesting models (e.g. the P $\Sigma M$ and most of its reductions). A promising alternative to the quantization of models in this class seems to be the use of the conserved non-local charges derived from (1). This approach has been pioneered by Lüscher [6] and developed further in

\footnotetext{
* Our conventions are: $\mu, \nu=0,1 ; \gamma^{00}=-\gamma^{11}=1 ; v_{ \pm}=v_{0} \pm v_{1} ; \epsilon^{01}=1$; $\left[\lambda^{a}, \lambda^{b}\right]=\mathrm{i} f^{a b c} \lambda^{c}$ with $f^{a b c}$ being the structure constants of $G$
} 
refs. $[7,8]$. In ref. [8] a general framework (called massive current algebras) has been set up, requiring in particular that the currents $I_{\mu}$ satisfying the quantum version of eqs. (1a, 1b) be Kac-Moody currents in the ulraviolet limit. Models with massive current algebras are quantum integrable i.e. they possess an infinite number of conserved (nonlocal) charges implying the absence of particle production and the factorizability of the $S$-matrix. To show that a given model has a massive current algebra is a non trivial problem requiring non-perturbative methods. In particular the P $\Sigma \mathrm{M}$ does not fit in this framework. We hope that our deformed sigma models with the commuting KMA's are in this class.

The principal sigma model in its standard form is obtained by solving eq. (1b) with $I_{\nu}=g^{-1} \partial_{\nu} g(g \in G)$ and then eq. (1a) is obtained from the Lagrangean

$$
S_{1}=\int d^{2} \xi \mathcal{L}_{1}=\frac{1}{2 e^{2}} \int d^{2} \xi \operatorname{Tr} \partial_{\mu} g^{-1} \partial^{\mu} g
$$

(Here we introduced a coupling constant, $e^{2}$, that could be scaled to one classically, but we prefer to keep it as it will play an important role in the quantum theory.) However in a completely analogous way we can solve eq. (1a) with $I^{\mu}=\epsilon^{\mu \nu} \partial_{\nu} \Phi$, then eq. (1b) for $\Phi$ is nothing but the equation following from the variation of

$$
S_{2}=\int d^{2} \xi \mathcal{L}_{2}=\frac{1}{2 e^{2}} \int d^{2} \xi\left(\partial_{\mu} \Phi^{a} \partial^{\mu} \Phi^{a}-\frac{1}{3} f^{a b c} \epsilon^{\mu \nu} \Phi^{a} \partial_{\mu} \Phi^{b} \partial_{\nu} \Phi^{c}\right)
$$

(This model was briefly discussed in [9].) The models described by $S_{1}$ and $S_{2}$ are physically different, since not only the $\mathrm{PB}$ structure of the currents is completely different but also - as we shall see later $-S_{2}$ describes a non asymptotically free theory, while the $\mathrm{P} \Sigma \mathrm{M}$ is asymtotically free.

In both models the Hamiltonian and the momentum can be written in terms of the currents as

$$
\begin{array}{lll}
H=\int d \xi^{1} \mathcal{H} & \mathcal{H}=\frac{1}{2}(V+\bar{V}) & V=\frac{1}{2 e^{2}} I_{+}^{a} I_{+}^{a} \\
P=\int d \xi^{1} \mathcal{P} & \mathcal{P}=\frac{1}{2}(V-\bar{V}) & \bar{V}=\frac{1}{2 e^{2}} I_{-}^{a} I_{-}^{a}
\end{array}
$$

but of course the Poisson algebra among the currents, $I_{\mu}^{a}$, following from the canonical PB's defined by $\mathcal{L}_{1}$ and $\mathcal{L}_{2}$ is different in the two cases.

In ref. [1] it has been pointed out, that in the case of the P $\Sigma \mathrm{M}$ the Poisson algebra of the currents admits an appropriate deformation depending on a continuous parameter such that the Hamiltonian equations of motion for the standard Hamiltonian, 
eq. (4a), are still identical to eqs. (1a, 1b). We generalise this observation slightly by allowing the presence of a Wess-Zumino term with a general coefficient $(\rho \neq 0)$ in $\mathcal{L}_{1}$, i.e. eqs. (1a, 1b) modified as

$$
\partial_{-} I_{+}=-\frac{1-\rho}{2}\left[I_{+}, I_{-}\right] \quad \partial_{+} I_{-}=\frac{1+\rho}{2}\left[I_{+}, I_{-}\right]
$$

and claim that eqs. (5) are the Hamiltonian equations for $H$ in eq. (4a) if the equal time PB's among the currents have the following general form:

$$
\begin{gathered}
\left\{I_{+}^{a}(\sigma), I_{+}^{b}(\hat{\sigma})\right\}=e^{2} f^{a b c}\left((1-\rho) I_{-}^{c}(\sigma)-[(1+2 x)-\rho(2 x-1)] I_{+}^{c}\right) \delta(\sigma-\hat{\sigma})+2 e^{2} \delta^{a b} \delta^{\prime}(\sigma-\hat{\sigma}) \\
\left\{I_{-}^{a}(\sigma), I_{-}^{b}(\hat{\sigma})\right\}=e^{2} f^{a b c}\left((1+\rho) I_{+}^{c}(\sigma)-[(1+2 x)+\rho(2 x-1)] I_{-}^{c}\right) \delta(\sigma-\hat{\sigma})-2 e^{2} \delta^{a b} \delta^{\prime}(\sigma-\hat{\sigma}) \\
\left\{I_{+}^{a}(\sigma), I_{-}^{b}(\hat{\sigma})\right\}=-e^{2} f^{a b c}\left((1-\rho) I_{+}^{c}(\sigma)+(1+\rho) I_{-}^{c}\right) \delta(\sigma-\hat{\sigma})
\end{gathered}
$$

Here $x$ is a new constant parameter that does not appear in eqs. (5), but whose presence in eq. (6) is allowed by the Jacobi identity. (Setting $\rho=0$ in eq. (6) we recover the Poisson algebra suggested in ref. [1].) Since in the Hamiltonian framework the specification of the fundamental PB's forms an essential part of the definition of the model, giving eq. (4-6) we defined a whole family of models depending on the new parameter $x$. Setting $x=1$ (and $\rho=0$ ) in eq. (6) we get the usual PB's of the principal sigma model while the $x=-1$ (and $\rho=0$ ) case of eq. (6) are identical to the canonical PB's of $I_{\mu}^{a}$ following from $\mathcal{L}_{2}$.

The very appealing property of the $x \neq \pm 1(\rho=0)$ models - that motivated the study of ref. [1] - is that at the PB level they admit two commuting Kac Moody algebras. Indeed if $x^{2}>1$ then - as a straightforward calculation shows - the linear combinations

$$
u_{ \pm}^{a}(\sigma)=\frac{1}{2 e^{2}}\left[-\frac{1}{x+1} I_{0}^{a}(\sigma) \pm \frac{1}{\sqrt{x^{2}-1}} I_{1}^{a}(\sigma)\right]
$$

form two commuting KM algebras with centres $k= \pm \frac{1}{2 e^{2}} \frac{1}{x+1} \frac{1}{\sqrt{x^{2}-1}}$ respectively, as a consequence of eq. (6). (If $x^{2}<1$ then we have to form complex linear combinations of $I_{0}^{a}$ and $I_{1}^{a}$ to get the KM currents $u_{ \pm}^{a} ; k$ in this case becomes $\pm \frac{1}{2 e^{2}} \frac{1}{x+1} \frac{1}{\sqrt{1-x^{2}}}$.) We see from these formulas that from the point of view of the KMA the $x= \pm 1$ models are certain singular limits of the generic case.

The study of these interesting models has been severely hindered by the fact that the Lagrangean found in ref. [1] was non local and not manifestly Lorentz invariant. Since for the $x= \pm 1, \rho=0$ cases local and manifestly Lorentz invariant Lagrangeans exist it seems natural to ask if this remains so for the general $x \neq \pm 1$ case, which 
in a certain sense, would interpolate between the $\mathrm{P} \Sigma \mathrm{M}$ and the theory defined by $\mathcal{L}_{2}$ (for $\rho=0$ at least). We are going to show that this is indeed the case at least for $G=S U(2)$.

As both $\mathcal{L}_{1}$ and $\mathcal{L}_{2}$ belong to the general class of Lagrangeans for bosonic sigma models with torsion we are going to work in this framework, i.e. we shall look for Lagrangeans of the form

$$
\mathcal{L}=\frac{1}{2 e^{2}} G_{A B}(X) \partial^{\mu} X^{A} \partial_{\mu} X^{B}-\frac{1}{2 e^{2}} B_{A B}(X) \epsilon^{\mu \nu} \partial_{\mu} X^{A} \partial_{\nu} X^{B}
$$

where $G_{A B}(X)$ is a metric on the underlying manifold and the antisymmetric tensor field $B_{A B}(X)$ is the torsion potential: $2 T_{A B C}=\partial_{A} B_{B C}+$ cyclic.

Together with the generalized sigma-model form of the Lagrangean (8) we make the following ansatz of the currents:

$$
I_{+}^{a}=Q_{A}^{a} \partial_{+} X^{A} \quad I_{-}^{a}=R_{A}^{a} \partial_{-} X^{A}
$$

and require that the Hamiltonian and momentum take the quadratic form (4) and that the KM-type current algebra relations (6) are consequences of the usual canonical Poisson brackets among the canonical variables $X^{A}$ and $\partial_{0} X^{A}$.

Note that both $Q_{A}^{a}$ and $R_{A}^{a}$ are vielbeins corresponding to the target space metric $G_{A B}$. It will turn out to be convenient to introduce the corresponding matrix valued 1-forms:

$$
-i Q_{A}^{a} d X^{A} \lambda^{a}=Q \quad-i R_{A}^{a} d X^{A} \lambda^{a}=R
$$

We will make the additional, but natural assumption that these 1-forms are related by a similarity transformation

$$
R=\epsilon g Q g^{-1}
$$

where $\epsilon^{2}=1$ and $g$ is a group-valued matrix.

Using the eqs. (1a, 1b) and the canonical structure that follows from the Lagrangean (8) with the definitions (9) we find that the requirements (4) and (6) are equivalent to a set of algebraic and differential equations satisfied by the Lie-algebra valued 1-form $Q$ and group-valued scalar matrix $g$ :

$$
\begin{aligned}
d Q & =A Q^{2}+\kappa \epsilon g^{-1} Q^{2} g \\
d R & =B R^{2}+\lambda \epsilon g R^{2} g^{-1} \\
T & =A \operatorname{Tr}\left\{Q^{3}\right\}+3 \kappa \epsilon \operatorname{Tr}\left\{g Q g^{-1} Q^{2}\right\} \\
& =-B \epsilon \operatorname{Tr}\left\{Q^{3}\right\}-3 \lambda \operatorname{Tr}\left\{g^{-1} Q g Q^{2}\right\}
\end{aligned}
$$


where $Q$ and $R$ are related by (11) and the torsion 3-form $T=T_{A B C} d X^{A} d X^{B} d X^{C}$ in (12) satisfies

$$
d T=0
$$

The constants $A, B, \kappa, \lambda$ are related to $x$ and $\rho$ in (6) as follows:

$$
\begin{array}{ll}
\kappa=\frac{1-\rho}{2} & A=-\lambda-2 x \kappa \\
\lambda=\frac{1+\rho}{2} & B=-\kappa-2 x \lambda
\end{array}
$$

and $\epsilon^{2}=1$.

The Lagrangeans $\mathcal{L}_{1}$ and $\mathcal{L}_{2}$ correspond to special solutions of (12-13). We do not know whether there are new, 'interpolating' solutions in the general case, but we have studied the simplest case of $S U(2)$ symmetry in detail.

We have taken the following special $S U(2)$ ansatz:

$$
\begin{aligned}
g & =\cos \phi+\sin \phi \cdot n \\
Q & =d \psi \cdot n+\alpha \cdot d n+\beta \cdot n d n
\end{aligned}
$$

where $n=i n^{a} \tau^{a} ; n^{a} n^{a}=1$ and $\psi, \phi, \alpha$ and $\beta$ are functions of the single 'radial' variable $r$. (This ansatz corresponds to an $S U(2)$ symmetric metric on the target space, the symmetry acting on $S^{2}$ spheres in the usual way.)

For this 3-dimensional target space (13) is satisfied automatically and (12) reduce to a set of ordinary differential equations for the unknown functions $\psi, \phi, \alpha$ and $\beta$, which can be solved completely. In addition to the cases $\mathcal{L}_{1}$ and $\mathcal{L}_{2}$, which are also included in (14), we find various other solutions. There are solutions with $x=1$ and $\rho=$ arbitrary or $x=$ arbitrary and $\rho=0$. (Within our $S U(2)$ ansatz there are no solutions with both parameters $x$ and $\rho$ taking arbitrary values, although this would be allowed by the algebra (6).) The former case corresponds to $\mathcal{L}_{1}$ with an additional WZ term. In our variables this Lagrangean is (with $r=w$ )

$$
\mathcal{L}=\frac{1}{2 e^{2}}\left\{\partial_{\mu} w \partial^{\mu} w+\cos ^{2} w \partial_{\mu} n^{a} \partial^{\mu} n^{a}-\rho(w+\sin w \cos w) \epsilon^{a b c} \epsilon^{\mu \nu} n^{a} \partial_{\mu} n^{b} \partial_{\nu} n^{c}\right\}
$$

The new solutions correspond to $\rho=0$ and after some linear rescaling they can be represented as

$$
\mathcal{L}=\frac{1}{2 e^{2}}\left\{\partial_{\mu} r \partial^{\mu} r+\frac{\beta_{0}}{x+1} \partial_{\mu} n^{a} \partial^{\mu} n^{a}+\frac{r-\alpha_{0}}{x+1} \epsilon^{a b c} \epsilon_{\mu \nu} n^{a} \partial_{\mu} n^{b} \partial_{\nu} n^{c}\right\}
$$


and

$$
\begin{array}{ll}
I_{+}^{a}=E_{+}^{a} & E=d r \cdot n+\alpha_{0} \cdot d n+\beta_{0} \cdot n d n \\
I_{-}^{a}=F_{-}^{a} & F=-d r \cdot n-\alpha_{0} \cdot d n+\beta_{0} \cdot n d n
\end{array}
$$

where

case a)

$$
\begin{array}{rlrl}
\phi=\text { const. } & \neq 0, \pi & x & =-\cos 2 \phi \\
\alpha_{0}=-\frac{1}{2} \operatorname{ctg} \phi & \beta_{0} & =\frac{1}{2}
\end{array}
$$

case b)

$$
\begin{gathered}
x^{2}>1 \quad r=\frac{1}{\sqrt{x^{2}-1}}\left(\frac{\pi}{2}-w\right) \\
\alpha_{0}=\frac{\sqrt{x^{2}-1}}{x+1} \frac{\sin w \cos w}{x+\cos 2 w} \quad \beta_{0}=\frac{\cos ^{2} w}{x+\cos 2 w}
\end{gathered}
$$

case c)

$$
x=1 \quad \alpha_{0}=\frac{r}{1+4 r^{2}} \quad \beta_{0}=\frac{2 r^{2}}{1+4 r^{2}}
$$

case d)

$$
\begin{array}{ccrl}
x^{2}<1 & \eta^{2}=1 & r & =\frac{w}{\sqrt{1-x^{2}}} \\
\alpha_{0}=\sqrt{\frac{1-x}{1+x}} \frac{\operatorname{sh} w \operatorname{ch} w}{\operatorname{ch} 2 w+\eta x} & \beta_{0}=\frac{1}{2} \frac{\operatorname{ch} 2 w+\eta}{\operatorname{ch} 2 w+\eta x}
\end{array}
$$

We note that in case b) it is natural to assume that $w$ is an angular variable. The usual argument about the single-valuedness of the exponentialized quantum action gives then the same quantization of the parameter $k$ (defined under (7)) as follows from the fact that it is the centre of the KM algebra.

It is interesting to note that we obtain the original P $\Sigma \mathrm{M}$ in a number of different ways. In addition to $\mathcal{L}_{1}$ the same equations of motion and canonical PB's can be obtained from (16) in case a) (with the special choice of $\phi=\pi / 2$ ) or as case c). These are inequivalent Lagrangean descriptions of the $\mathrm{P} \Sigma \mathrm{M}$ since there is no local transformation of the field variables that would transform these cases of (16) to $\mathcal{L}_{1}$. We also note that the conformal WZNW model, which corresponds to (15) with $\rho=1$, can also be obtained from (16), case b) in the limit $x \rightarrow \infty, e^{2} x^{2} \rightarrow$ const.

The next important question concerns the quantum integrability of our models. A promising possibility would be to show the existence of massive current algebras of ref. [8] i.e. the existence of conserved non-local charges. This problem requires further study. Here we merely want to show, that an appropriate modification of Polyakov's 
heuristic argument for the existence of a local higher spin conserved quantity in the quantized versions of $O(N)$ sigma models [10] applies to the entire family of our $S U(2)$ models as well. Since this quantity is the quantum descendant of a local non polynomial classical quantity [4] we derive this latter first.

Classically, as a consequence of eqs. (1a, 1b), we have

$$
\partial_{-} \operatorname{Tr} I_{+}^{2}=0
$$

This equation (together with its chiral partner) expresses the vanishing of the trace of the energy momentum tensor thus the classical conformal invariance of our models. It is not difficult to establish that in the case of $S U(2)$, exploiting the specific properties of $\epsilon^{a b c}$ together with eqs. (1), but without ever using eq. (6), we get

$$
\partial_{-} \operatorname{Tr}\left(\partial_{+} I_{+}\right)^{2}=-\partial_{+}\left\{\frac{1}{2} \operatorname{Tr} I_{+}^{2} \operatorname{Tr} I_{+} I_{-}\right\}+\frac{3}{2} \operatorname{Tr} I_{+}^{2} \partial_{+}\left(\operatorname{Tr} I_{+} I_{-}\right)
$$

Introducing

$$
U=\operatorname{Tr}\left(\partial_{+}\left[\frac{I_{+}}{\sqrt{\operatorname{Tr} I_{+}^{2}}}\right]\right)^{2}, \quad V=\frac{\operatorname{Tr} I_{+} I_{-}}{\sqrt{\operatorname{Tr} I_{+}^{2}}}
$$

we can indeed write this equation in the form of a non polynomial conservation equation $\partial_{-} U=\partial_{+} V$. However the essential part of Polyakov's argument is the assumption about the quantum modifications of eqs. (18), (19) generated by the conformal anomaly. According to this assumption (which is implicitly based on the existence of a local Lagrangean) the anomalous terms appearing in eqs. (18), (19) are local operators with conformal dimensions, spins and global quantum numbers determined by the classical equations. Supplementing these ideas with the additional assumption that all these operators can be constructed from $I_{+}, I_{-}$we find the quantum version of eq. (18) as

$$
\partial_{-} \operatorname{Tr} I_{+}^{2}=\mu\left(e^{2}, x\right) \partial_{+} \operatorname{Tr} I_{+} I_{-}
$$

Furthermore, using the equations of motion, we can show that the sum of all possible anomalous terms appearing in eq. (19) can be written as the sum of total $\partial_{+}$derivatives plus a term proportional to $\operatorname{Tr} I_{+}^{2} \partial_{+}\left(\operatorname{Tr} I_{+} I_{-}\right)$. Thus integrating the quantum version of eq. (19) over $x^{+}$and dropping the surface terms we get

$$
\partial_{-} \int d x^{+} \operatorname{Tr}\left(\partial_{+} I_{+}\right)^{2}=(3 / 2+\nu) \int d x^{+} \operatorname{Tr} I_{+}^{2} \partial_{+}\left(\operatorname{Tr} I_{+} I_{-}\right)
$$


(In eqs. (20), (21) $\mu$ and $\nu$ are some numerical factors related to the conformal anomaly.) Substituting $\partial_{+}\left(\operatorname{Tr} I_{+} I_{-}\right)$from eq. (20) we indeed find

$$
\partial_{-} Q=0, \quad Q=\int d x^{+}\left(\operatorname{Tr}\left(\partial_{+} I_{+}\right)^{2}-(1 / 2 \mu)(3 / 2+\nu)\left(\operatorname{Tr} I_{+}^{2}\right)^{2}\right)
$$

The exsistence of this spin three conserved quantity, $Q$, would, according to the standard argument $[10,11]$ guarantee the absence of particle production.

The advantage of using Lagrangeans that are special cases of the general framework of sigma-models with torsion is that we can use the renormalizability of these models. Actually, it is not completely trivial that our models are renormalizable since although the general models are [12], we have to show that the neccessary counter terms, which are known in the general case [13], can be obtained as renormalizations of our coupling parameters $e^{2}$ and $x$ (together with a non-linear renormalization of the 'radial' field $r$ ). At least to one-loop order, this is indeed the case, and we obtain, using the general results in the dimensional regularization scheme [13], that under the renormalization group transformation of the substraction point $\mu \rightarrow e^{t} \mu$ the renormalized couplings $\alpha=e^{2} / \pi$ and $x$ satisfy the following RG equations

$$
\begin{aligned}
\dot{\alpha} & =(1-2 x) \alpha^{2}+\mathcal{O}\left(\alpha^{3}\right) \\
\dot{x} & =\left(x^{2}-1\right) \alpha+\mathcal{O}\left(\alpha^{2}\right)
\end{aligned}
$$

(23) is valid for all cases of the new Lagrangeans (16), and it also applies to $\mathcal{L}_{1}$ and $\mathcal{L}_{2}$ (which correspond to the cases $x=1$ and $x=-1$, respectively).

From (23) we see that $x^{2}=1$ is a special fixed line in the space of the two couplings. For $x=1$ we recover the well-known asymptotically free behaviour of the $\mathrm{P} \Sigma \mathrm{M}$ coupling, whereas for $x=-1$ we find that the model described by $\mathcal{L}_{2}$ is not asymptotically free. (This was first pointed out in ref. [14].) Thus these two models describe very different physics, as mentioned earlier.

We note that the combination $1 / 2 \pi k=\alpha(x+1) \sqrt{x^{2}-1}$, which must be quantized, is an invariant of the RG trajectories defined by (23). However, our perturbative results, which are obtained for fixed $x$ and small $\alpha$, are only valid for large $k$.

The most interesting trajectories correspond to the $x>1$ case. In this case the trajectories lead to the UV fixed point $e^{2}=0, x=\infty$. More precisely, $x \rightarrow \infty$, while $e^{2} x^{2} \rightarrow$ const. (since $k$ is fixed). Thus our models tend to the conformal WZNW model at their UV fixed points.

We have also calculated the behaviour of the current-current 2-point function in perturbation theory for the properly normalized Noether current

$$
N_{\mu}^{a}=\frac{-1}{e^{2}(x+1)} I_{\mu}^{a}
$$


In Euclidean space, after renormalization, we find

$$
\left\langle N_{\mu}^{a}(x) N_{\nu}^{b}(y)\right\rangle=\frac{\delta^{a b}}{\pi} \int \frac{d^{2} p}{2 \pi} e^{-i p(x-y)}\left(\frac{p_{\mu} p_{\nu}}{p^{2}}-\delta_{\mu \nu}\right) I(p)
$$

where

$$
I(p)=\frac{1}{\alpha(x+1)^{2}}+\frac{1}{2(x+1)^{2}}\left(\ln \frac{p^{2}}{\mu^{2}}+\text { const. }\right)+\mathcal{O}(\alpha)
$$

Using (26) and the renormalization group we find that for the trajectories ending at the WZNW fixed point

$$
I(p) \rightarrow \text { const. }
$$

so from this point of view our models show fermionic behaviour. At this point we would like to remark that the result in (26) may be considered as a first step to calculate the operator product expansion of two currents. If in the RG eqs. (23) the higher order corrections can be shown to go zero faster than the leading terms then one can establish the existence of a massive current algebra of ref. [8] by perturbation theory. This would be similar to the calculation of Lüscher [6] who used asymptotic freedom to find the short distance expansion of the product of two currents.

In conclusion in this letter we derived local Lagrangeans for a new family of $S U(2)$ invariant sigma models admitting two commuting KMA's classically. We have established their one loop renormalizability and have given two independent arguments in favour of the quantum integrability of the $x>1$ subset.

Acknowledegements We would like to thank M. Niedermaier and C. Zachos for calling our attention to ref. [1] and ref. [14] respectively. This work was partially supported by the Hungarian National Science and Research Foundation (Grant No. 2177 and No. 1815). 


\section{References}

[1] S. G. Rajeev, Phys. Lett. B217 (1989) 123

[2] A. Polyakov and P. B. Wiegmann, Phys. Lett. B131 (1983) 121

[3] L. D. Faddeev and N. Yu. Reshetikin, Ann. Phys. 167 (1986) 227

[4] K. Pohlmeyer, Comm. Math. Phys. 46 (1976) 207

[5] L. D. Faddeev in Les Houches lectures (1982) on Recent Advances in Field Theory and Statistical Mechanics, eds. J. B. Zuber and R. Stora (North Holland, Amsterdam, 1984)

[6] M. Lüscher Nucl. Phys. B135 (1978) 1

[7] H. De Vega, H. Eichenherr, J. M. Maillet, Nucl. Phys. B240 (1984) 377, Comm. Math. Phys. 92 (1984) 507

[8] D. Bernard, Comm. Math. Phys. 137 (1991) 191

[9] V. E. Zakharov and A. V. Mikhailov, Sov. Phys. JETP 47 (1978) 1017

[10] A. Polyakov, Phys. Lett. B72 (1977) 224

[11] A. B. Zamolodchikov and A. B. Zamolodchikov, Ann. Phys. 120 (1979) 253

[12] D. Friedan, Ann. Phys. 163 (1985) 318 for further references see the recent review V. V. Belokurov and D. I. Kazakov, Sov. J. Part. Nucl. 23 (1992) 577

[13] H. Osborn, Ann. Phys. 200 (1990) 1

[14] Ch. Nappi, Phys. Rev. D21 (1980) 418 